\title{
Playing the Goblin Post Office game improves movement control of the core: A case study.
}

\author{
Gabor J Barton, Malcolm B Hawken, Richard J Foster \\ Research Institute for Sport and Exercise Sciences \\ Liverpool John Moores University \\ Liverpool, United Kingdom. \\ email: g.j.barton@ljmu.ac.uk
}

\author{
Gill Holmes \\ North West Movement Analysis Centre \\ Alder Hey Children's NHS Foundation Trust \\ Liverpool, United Kingdom.
}

\author{
Penny B Butler \\ The Movement Centre \\ Oswestry, United Kingdom.
}

\begin{abstract}
Movement function of the core (trunk and pelvis) can be improved in cerebral palsy, potentially leading to benefits which transfer to activities of daily living. A single child with $C P$ diplegia played our custom made game which runs on the CAREN system. Three playing postures gradually introduced more and more joints in the legs to be controlled. Vicon cameras tracked trunk and pelvic rotations which drove a dragon towards envelope targets. Forward speed of the game was adjusted by an adaptive algorithm leading to a maximum settled speed for the various conditions. Results showed that core control improved after the six week training period. The trunk was better controlled than the pelvis, sideways rotations were better controlled than fore-aft rotations of body segments, and single plane rotations were more efficient than cross-plane rotations of the core. The quantifiable improvements suggest a good potential for our technique to improve core control which is a prerequisite for good movement control of the legs and arms.
\end{abstract}

Keywords - cerebral palsy; movement training; core control; virtual rehabilitation

\section{INTRODUCTION}

Traditionally the core of the body (pelvis and trunk) has been regarded as a "passenger unit" carried by the lower extremities which are termed the "locomotor unit" [1] suggesting a passive role for the pelvis and trunk in gait. There is however evidence supporting a more active role for the pelvis and trunk. Responses triggered by balance perturbations involve trunk, neck and thigh muscles which contract at the same time, or even before the activation of muscles around the ankle joint [2-5]. In prospective studies [6,7] reduced strength and impaired proprioception around the core could be associated with increased risk of injuries, specifically around the knee, suggesting that control over the core is a prerequisite for efficient and effective use of the legs.

Cerebral palsy $(\mathrm{CP})$ is a neuro-musculo-skeletal disorder caused by damage to the immature brain around birth. It has an incidence of 1 in 400 live births [8]. The treatment of children with CP focuses mainly on secondary abnormalities (altered

This project was funded by The WellChild Trust and Alder Hey Children's NHS Foundation Trust. bone and muscle growth) and avoids addressing the primary abnormalities characterizing $\mathrm{CP}$ which include loss of selective muscle control, muscle imbalance and deficient equilibrium reactions [9]. These abnormalities affecting the pelvis and trunk lead to reduced stability and control of the core, which impairs movement control of distal body segments (arms and legs) leading to inefficient performance of activities of daily living.

In spite of the primary damage to the central nervous system in $\mathrm{CP}$, global motor function can be improved by controlled exercises that take advantage of the brain's capacity to reorganize in targeted areas (neuroplasticity). Reactive balance training of children with $\mathrm{CP}$ on a movable platform led to improvement of muscle responses, and the quantifiable benefits were carried over for one month after training [10]. Balance training improved stance and gait leading to a more symmetrical walking pattern in a group of children with hemiplegic CP [11].

The clinical application of virtual reality (VR) is expanding dramatically. It is a promising area for the application of new technologies in movement rehabilitation, underpinned by a growing number of published studies supporting the feasibility and benefits of virtual rehabilitation [12]. Research efforts in the area of movement rehabilitation using VR have focused mainly on training the arms and legs [13-17]. The aim of this study was to evaluate the effects of a six week training programme using a custom made VR game developed to train movement control of the trunk and pelvis. Results from a single child with CP diplegia are presented here.

\section{MethodS}

\section{A. Participant and training/testing protocol}

One boy with CP diplegia (age: 10 years; height: $1.34 \mathrm{~m}$; mass: $36 \mathrm{~kg}$ ) trained on a custom-made computer game called the "Goblin Post Office" (GPO) for a period of six weeks, twice a week, for 30 minutes in each session. The child had no previous history of surgical intervention and received no 
conventional physiotherapy or core specific rehabilitation which might influence VR training. He maintained his regular physical activity in PE lessons and swimming with family throughout the study. Prior to and shortly after the six week training period, the child's performance was measured using the GPO to establish whether there were any changes in response to VR training.

\section{B. The Goblin Post Office game}

A Computer Assisted Rehabilitation Environment (CAREN System, Motek Medical, Amsterdam, The Netherlands) was used to create the interactive game. The CAREN system consists of an 8-camera Vicon 612 optoelectronic motion capture system (Oxford Metrics, Oxford, UK), a six degreesof-freedom moving platform and video projection. Components of the system are controlled by the CAREN D-Flow software (Fig. 1). The aim of the "Goblin Post Office" (GPO) was to use movement of the trunk or pelvis to navigate a dragon through a virtual cave consisting of alternating straight segments and $90^{\circ}$ bends. The horn protruding from the dragon's head was used to burst targets that contain virtual letters. Two clusters of three retro-reflective markers, each forming a triangle, were placed on the trunk and on the pelvis to track the segments during game-play.

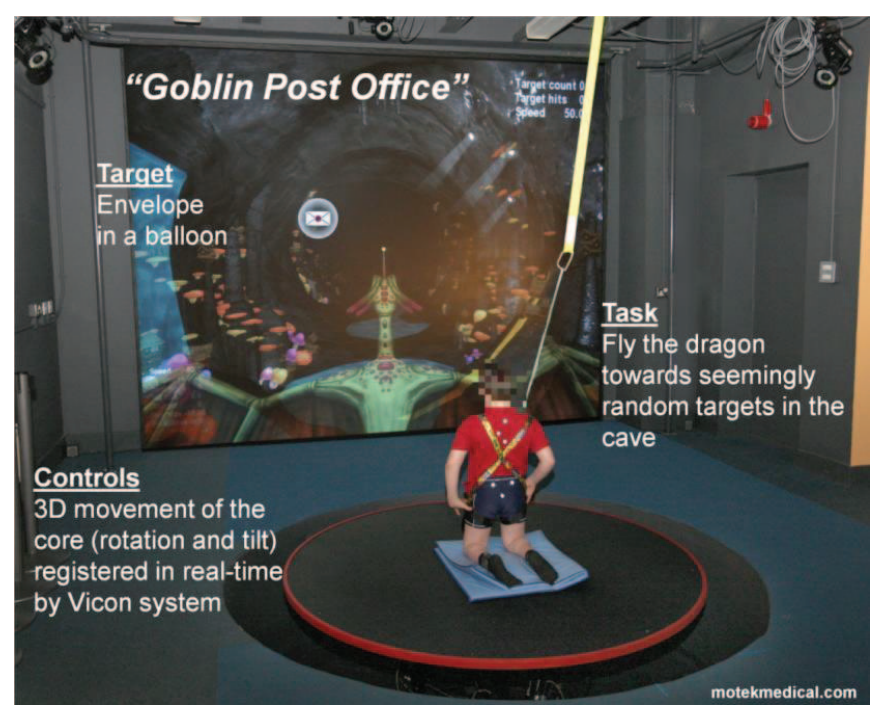

Figure 1: The Goblin Post Office game as played by the participant. Note the infra-red cameras mounted on the walls, tracking positions of the two marker clusters on the trunk and pelvis.

\section{Control Schemes}

To steer the dragon right or left in the GPO required rotation of the trunk or pelvis in the transverse plane, whilst anterior and posterior tilt in the sagittal plane steered down and up respectively. Angular displacement of the body segment controlled speed of flight, not displacement, in the appropriate direction. In a familiarization session the child moved the dragon right, left, up and down without any forward progression, by rotating and tilting the trunk and then the pelvis in order to understand the mapping of segmental movements to motion in the game. At first, the participants used rotation or tilt of the trunk or pelvis to play the game. This meant the dragon could only move horizontally or vertically in order to navigate towards the virtual targets which appeared unpredictably in a horizontal or vertical row. After familiarization with cross-plane movements driven by simultaneous rotation and tilt, the virtual targets appeared in unpredictable locations requiring navigation in oblique directions. Targets were presented in blocks of 8 in the straight segments of the cave. Movements between targets were always the same length, and in one of eight possible directions.

\section{Levels of difficulty}

The theory of Targeted Training [18,19] aims to simplify movement tasks by stabilizing the body below the level of the targeted joint. We used this concept to define three levels of difficulty of core control (Fig 2.). The purpose of the first level, kneel sitting, was to stabilize the leg and pelvis, thereby focusing on movement of the trunk only. At the second level the child assumed a high kneeling position which introduced additional control of the hip and knee joints while stabilizing the shank and foot segments during game-play. The third level was standing which required coordinated movement of all joints and body segments involved in upright standing. At all levels, the child began with single plane movements of the trunk segment, followed by cross-plane movements, eventually controlling the game with their pelvis. The criterion for progressing to the next level was either reaching a settled level of performance or reaching a time limit.
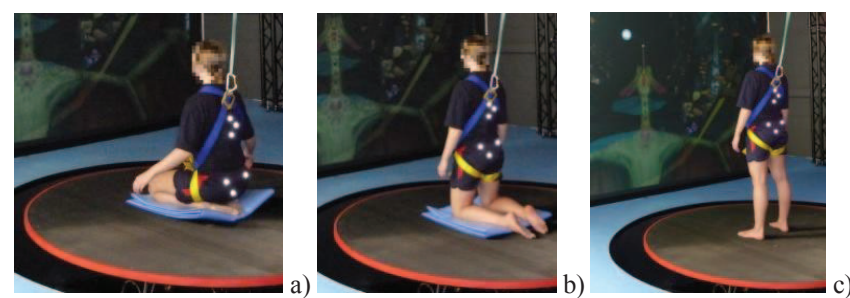

Figure 2: The three postures of a) kneel sitting, b) high kneeling, and c) standing which introduced progressively more joints and segments making the control of trunk and pelvis movements more and more difficult.

\section{E. Measure of performance during game-play}

For the game to remain interesting the level of difficulty needed to match the subject's performance, which varied over time and between subjects. The GPO game incorporates an adaptive algorithm (PEST) [20] which varies game difficulty, but in addition provides a method of measuring performance. The algorithm adjusts the forward speed of the virtual dragon during game-play to vary game difficulty. Speed increases in response to a successful collision with a target, thus increasing difficulty for the subject, speed reduces following a miss. The algorithm also adjusts the size of speed increments automatically over time, and so speed (and hit rate) converge towards a settled value at which increments were minimum, representing stable performance. A custom MATLAB (MathWorks, Natick MA, USA) program was used to determine the maximum settled speed (MSS), defined as the highest mean speed with the smallest speed variance over a straight segment of the virtual cave. 


\section{RESULTS}

\section{A. Speed profiles and the maximum settled speed}

The game starts at $50 \mathrm{~m} / \mathrm{s}$ forward speed of the dragon (in game-scale metres) and then the speed varies as a result of the speed adjustment algorithm. A typical speed profile recorded during game-play is shown in Fig. 3 illustrating how the game speed settles over time.

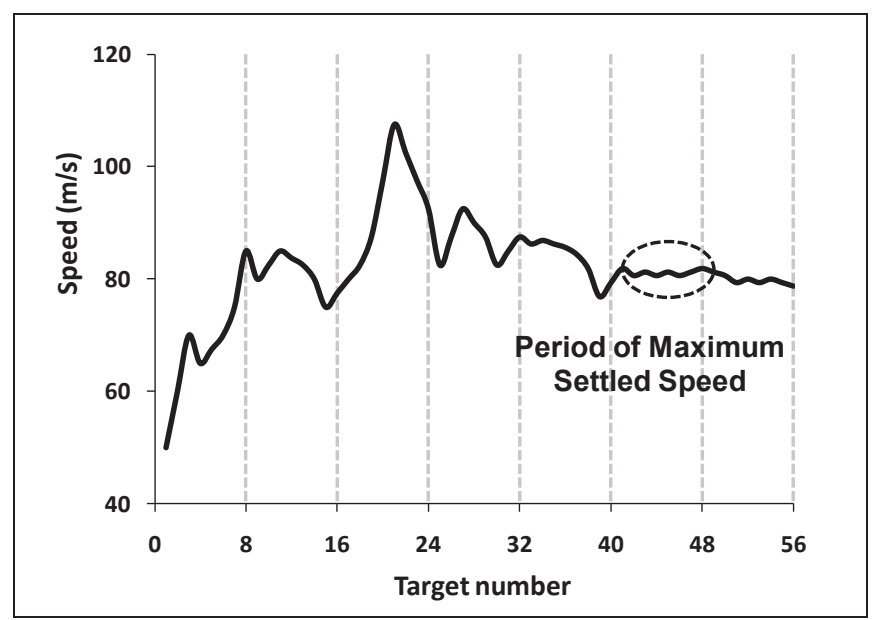

Figure 3. A typical example of a speed profile while playing the Goblin Post Office game driven by rotation of the trunk. Game speed changes dynamically as a function of target hit ratios. The maximum settled speed was reached in the $6^{\text {th }}$ straight section after popping the $40^{\text {th }}$ balloon.

\section{B. Single plane compared to cross-plane motion of the trunk}

The maximum settled speeds (MSS) reached using single and dual plane motion at pre- and post-training suggest that greater speeds were achieved in the GPO game when using either trunk rotation or trunk tilt alone, rather than rotating and tilting the trunk simultaneously. This was the case for all levels of the GPO game in kneel sitting, high kneeling and standing (Fig. 4).

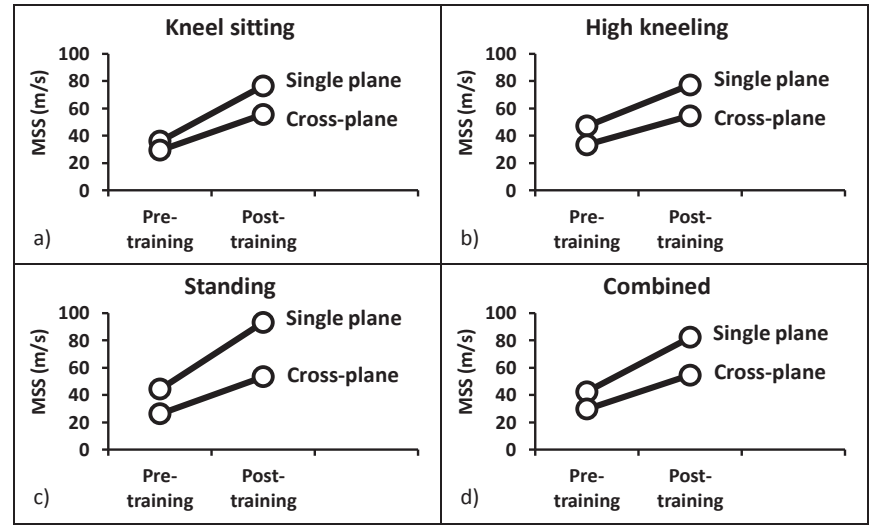

Figure 4. Comparison of single plane and cross-plane maximum settled speed (MSS) values in a) kneel sitting, b) high kneeling, c) standing, and d) averaged, before and after training on the Goblin Post Office game.

\section{Trunk rotation compared to trunk tilt}

Performance peaked when steering the dragon using trunk rotation rather than trunk tilt, at all levels of the game separately (kneel sitting, high kneeling, standing), and with a mean difference of $6.7 \mathrm{~m} / \mathrm{s}$ during pre-training, and $3.0 \mathrm{~m} / \mathrm{s}$ post-training when averaging the values across levels (Fig. 5).

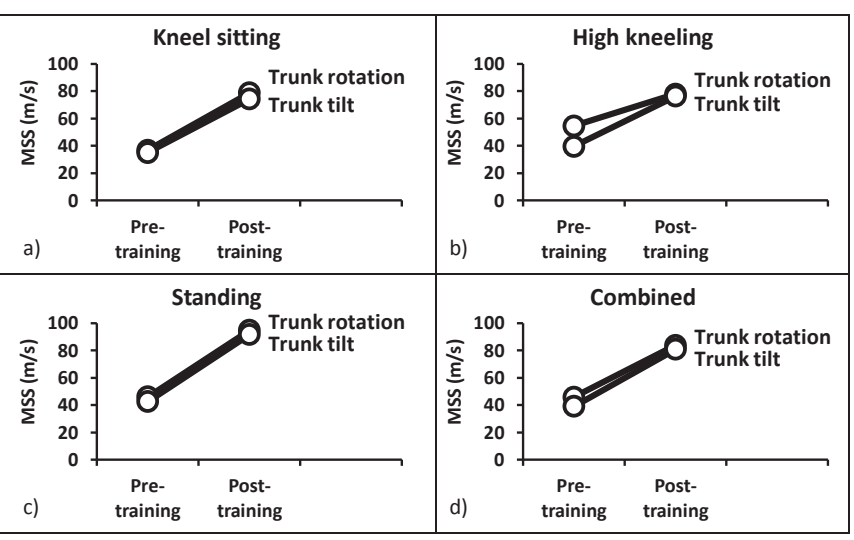

Figure 5. Comparison of maximum settled speed (MSS) achieved with rotation compared to tilt of the trunk in a) kneel sitting, b) high kneeling, c) standing, and d) averaged, before and after training.

\section{Trunk rotation compared to pelvis rotation}

Higher values of MSS were achieved using trunk rotation to steer the dragon in both high kneeling and standing (Fig. 6). Combining the results of high kneeling and standing, mean MSS of $50.3 \mathrm{~m} / \mathrm{s}$ (pre-training), and $86.4 \mathrm{~m} / \mathrm{s}$ (post-training) was found when using trunk rotation, compared to $38.9 \mathrm{~m} / \mathrm{s}$ (pre-training) and $60.8 \mathrm{~m} / \mathrm{s}$ (post-training) when using pelvic rotation.

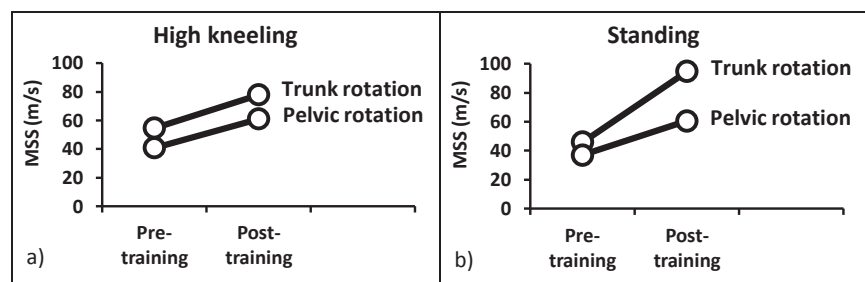

Figure 6. Comparison of maximum settled speed (MSS) values reached with trunk and pelvis rotation in a) high kneeling and b) standing, before and after training. Note that pelvic rotation was not possible in kneel sitting.

\section{E. Comparison of performance at three GPO levels pre-and post-training}

In kneel sitting, there was a significant difference in performance from pre- to post-training $\left(\mathrm{t}_{2}=-7.442, \mathrm{p}=0.018\right)$, evidenced by an increase in mean MSS from $33.8 \pm 4.0 \mathrm{~m} / \mathrm{s}$ to $69.6 \pm 12.3 \mathrm{~m} / \mathrm{s}$ across all levels (Fig. 7a). There was a significant difference between pre- and post-training in high kneeling $\left(\mathrm{t}_{3}=-6.596 \mathrm{p}=0.007\right)$, shown by an increase in mean MSS from $42.1 \pm 9.0 \mathrm{~m} / \mathrm{s}$ to $67.5 \pm 11.5 \mathrm{~m} / \mathrm{s}$ (Fig. 7b). In standing there was also a significant difference between preand post-training $\left(\mathrm{t}_{3}=-5.442 \mathrm{p}=0.012\right)$, demonstrated by an increase in mean MSS from $37.9 \pm 8.7 \mathrm{~m} / \mathrm{s}$ to $75.2 \pm 21.2 \mathrm{~m} / \mathrm{s}$ (Fig. 7c). 


\section{F. Overall comparison of performance between pre- and post-training}

MSS increased at all levels of the GPO game, with a mean change in speed of $32.6 \pm 11.0 \mathrm{~m} / \mathrm{s}$. The largest increase was demonstrated during standing whilst using the trunk to drive the game $(49.3 \mathrm{~m} / \mathrm{s})$ (Fig. $7 \mathrm{~d})$.

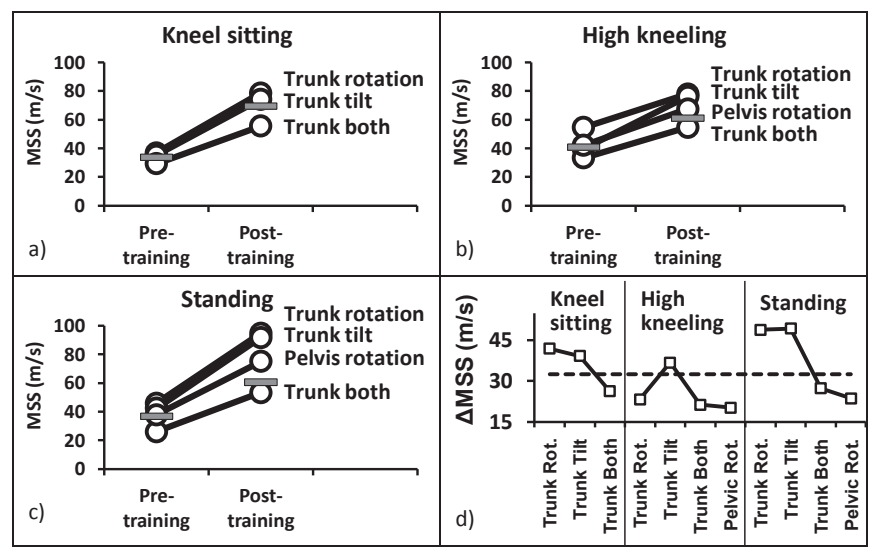

Figure 7. The changes of maximum settled speed (MSS) between pre- and post-training when playing the game in a) kneel sitting, b) high kneeling, and c) standing. Short horizontal bars indicate the mean MSS pre- and posttraining. Differences of MSS between pre- and post-training for all conditions are shown in d) where the dotted line shows the mean difference across all conditions.

\section{DISCUSSION}

The boy completed the six weeks training without missing any of the sessions. He received minimal encouragement from the experimenters and parents and so his motivation was maintained mostly by the varying difficulty of the game set by the adaptive speed adjustment algorithm.

A systematic analysis of the maximum settled speeds reached while playing the game leads to a number of observations. In addition to confirming the effect of game training, interpretation of these observations reveals several underlying mechanisms which improve our understanding of how the trunk and pelvis are used by a child with CP diplegia in a real-time biofeedback setting.

\section{A. Single plane movements are better controlled than cross- plane movements.}

The quasi-random positions of balloon targets were such that the child had to navigate by rotating and tilting the body segment (trunk or pelvis) at different ratios resulting in oblique flight paths of the dragon. This combined task of simultaneously controlling segmental movement across principal planes appeared to be more difficult than single plane movements in the transverse or sagittal planes. In addition, the MSS improved pre- to post-training more in single plane movements than in cross-plane movements. Gait involves small range movements of the pelvis but these occur simultaneously combining pelvic tilt, obliquity and rotation and so the inclusion of cross-plane movements of the core in game training might be expected to translate to daily living activities better than single plane movements.

\section{B. Trunk rotation is controlled better than trunk tilt.}

Reduced control of segmental tilt in comparison to rotation may occur due to the destabilizing effect of the body's centre of gravity as it translates outside the base of support, whilst rotation is likely to result in less displacement of the whole body's centre of gravity. Another factor may be that retraction of one side is unambiguously mapped to steering in the same direction but anterior tilt may be expected to produce either upward or downward movement, depending on the participant's previous experience of computer games. In addition, the range of motion when rotating a segment in the transverse plane is symmetrical but is asymmetrical for pelvic tilt with greater range of anterior tilt than posterior tilt in the sagittal plane. Gains mapping tilt of the segment to vertical speed of the dragon were set so that the child was able to reach all targets using the more limited posterior tilt range but as a result only a reduced section of the full range of anterior tilt was used. Such anatomically determined asymmetry might have posed an added level of complexity resulting in lower MSS when using tilt.

\section{Trunk rotation is controlled better than pelvic rotation.}

Higher MSS values were reached when using rotation of the more cephalic trunk segment in comparison to the more caudal pelvic segment. This finding confirms the theories underlying Targeted Training [18,19] with development of trunk control progressing segmentally in a cephalo-caudal direction in typically developing infants [21]. Control has also been shown to improve cephalo-caudally with Targeted Training in children with CP $[22,23]$. Thus the observation in this study of reduced movement control of the pelvis compared to the trunk is not unexpected. The inability of the child to use pelvic tilt to drive the game sufficiently well (ruling out comparison of trunk tilt and pelvic tilt) further suggests reduced control at pelvic level.

\section{The ranking order remains unchanged for body segments and planes of movement pre-to post-training.}

With only one exception, none of the lines in the above figures cross confirming that the constraints determining the level of control of a certain segment in a specific task change in parallel.

\section{E. The highest maximum settled speed was reached in high kneeling pre-training but in standing post-training.}

After training the child performed best in the most complex task of standing where all segments of the body required simultaneous control. Such an improvement is likely to confer greater ability in activities of daily living.

\section{F. The maximum settled speed improved as a result of playing the GPO game.}

Movement control of both the pelvis and trunk segments improved in kneel sitting, high kneeling and standing with either single or dual plane motions of the targeted segment controlling the game. The statistically significant differences confirm that a six week training programme resulted in measurable improvements at all levels. 


\section{CONCLUSIONS}

Our study confirms the beneficial effects of playing a custom made computer game on movement control of the trunk and pelvis in a child with cerebral palsy. The maximum settled speed algorithm provides a measure of motor performance and enables monitoring of change over time. Changes in the MSS suggest that the trunk is better controlled than the pelvis, rotation is better controlled than tilt, and single plane movements are executed with more control than cross-plane movements. Improvement of motor performance was highest in standing confirming the potential of this technique to improve activities of daily living.

\section{REFERENCES}

[1] J. Perry, Gait Analysis: Normal and Pathological Function. Thorofare, NJ: SLACK- Inc., 1992.

[2] J. H. Allum, B. R. Bloem, M. G. Carpenter, M. Hulliger, and M. Hadders-Algra, "Proprioceptive control of posture: a review of new concepts" Gait Posture, vol. 8(3), pp. 214-242, 1998.

[3] E. A. Keshner, M. H. Woollacott, and B. Debu, "Neck, trunk and limb muscle responses during postural perturbations in humans." Exp. Brain Res., vol. 71(3), pp. 455-466, 1988.

[4] J. H. Allum, F. Honegger, and H. Acuña, "Differential control of leg and trunk muscle activity by vestibulo-spinal and proprioceptive signals during human balance corrections." Acta Otolaryngol., vol. 115(2), pp. 124-129, 1995

[5] H. Forssberg H, and H. Hirschfeld, "Postural adjustments in sitting humans following external perturbations: muscle activity and kinematics.” Exp. Brain Res., vol. 97(3), pp. 515-527, 1994.

[6] D. T. Leetun, M. L. Ireland, J. D. Willson, B. T. Ballantyne, and I. M. Davis, "Core stability measures as risk factors for lower extremity injury in athletes.” Med. Sci. Sports Exerc., vol. 36(6), pp. 926-934, 2004.

[7] B. T. Zazulak, T. E. Hewett, N. P. Reeves, B. Goldberg, and J. Cholewicki, "The effects of core proprioception on knee injury: a prospective biomechanical-epidemiological study.” Am. J. Sports Med., vol. 35(3), pp. 368-373, 2007.

[8] L. A. Koman, B. P. Smith, and J. S. Shilt, "Cerebral palsy.” Lancet, vol. 363(9421), pp. 1619-1631, 2004

[9] J. R. Gage, and T. F. Novacheck, "An update on the treatment of gait problems in cerebral palsy.” J. Pediatr. Orthop. B, vol 10(4), pp. 265 274, 2001.

[10] M. H. Woollacott, A. Shumway-Cook, S. Hutchinson, M. Ciol, R. Price, and D. Kartin, "Effect of balance training on muscle activity used in recovery of stability in children with cerebral palsy: a pilot study." Dev. Med. Child Neur., vol. 47(7), pp. 455-461, 2005.

[11] A. Ledebt, J. Becher, J. Kapper, R. M. Rozendaalr, R. Bakker, and I. C. Leenders, "Balance training with visual feedback in children with hemiplegic cerebral palsy: effect on stance and gait." Motor Control, vol. 9(4), pp. 459-468, 2005.

[12] F. D. Rose, B. M. Brooks, and A. A. Rizzo, "Virtual reality in brain damage rehabilitation: review." Cyberpsychol. Behav., vol. 8, pp. 241 262; discussion pp. 263-271, 2005.

[13] J. E. Deutsch, J. Latonio, G. C. Burdea, and R. Boian, "Post-stroke rehabilitation with the Rutgers Ankle system: A case study." PresenceTeleoperators and Virtual Environments, vol. 10(4), pp. 416-430, 2001.

[14] A. S. Merians, D. Jack, R. Boiau, M. Tremaine, G. C. Burdea, and S. V. Adamovich, "Virtual reality-augmented rehabilitation for patients following stroke.” Physical Therapy, vol. 82(9), pp. 898-915, 2002.

[15] C. Bryanton, J. Bosse, M. Brien, J. McLean, A. McCormick, and H. Sveistrup, "Feasibility, motivation, and selective motor control: Virtual reality compared to conventional home exercise in children with cerebral palsy." Cyberpsychol. Behav., vol 9(2), pp. 123-128, 2006.

[16] A. S. Merians, H. Poizner, R. Boian, G. Burdea, and S. Adamovich, "Sensorimotor training in a virtual reality environment: Does it improve functional recovery poststroke?” Neurorehab. Neur. Rep., vol. 20(2), pp. 252-267, 2006

[17] S. H. You, S. H. Jang, Y. H. Kim, M. Hallett, S. H. Ahn, and Y. H. Kwon, "Virtual reality-induced cortical reorganization and associated locomotor recovery in chronic stroke - An experimenter-blind randomized study." Stroke, vol. 36(6), pp. 1166-1171, 2005.

[18] P. B. Butler, and R. E. Major, "The learning of motor control. Biomechanical considerations.” Physiotherapy, vol 78(1), pp. 1-6, 1992.

[19] R. E. Major, G. R. Johnson, and P. B. Butler, "Learning motor control in the upright position: A mechanical engineering approach.” Proc. Inst. Of Mech. Eng., vol. 215, pp. 315-323, 2001.

[20] M. M. Taylor, and C. D. Creelman, "PEST: Efficient estimates on probability functions.” J. Acoust. Soc. Am., vol. 41(4A), pp. 782-787, 1967.

[21] S. Saavedra, and M. H. Woollacott, "Development of trunk control in typically developing children and children with cerebral palsy" Proc. of meeting of the Collaborative Project on Developmental Neurology, Groningen, The Netherlands, pp. 54-55. 2010.

[22] P. B. Butler, "A preliminary report on the effectiveness of trunk targeting in achieving independent sitting balance in children with cerebral palsy." Clinical Rehabilitation, vol. 12(4), pp. 281-293, 1998.

[23] S. Saavedra, and M. H. Woollacott, "Effects of segmental training on trunk control in children with neuromotor disability: a pilot study." Proc. of meeting of the Collaborative Project on Developmental Neurology, Groningen, The Netherlands, pp. 55-56, 2010. 